\title{
A Different Approach for Spatial Prediction and Transform Using Video Image Coding
}

\author{
D.Menaka, Student of M.E (VLSI DESIGN), Dr.C.N Marimuthu \\ Dean/ECE Nandha Engineering College,Erode-52.
}

\begin{abstract}
A novel approach to jointly optimized spatial prediction the choice of the subsequent transform in video and image compression. In this paper we improve the performance, reduce cost and high complexity. In proposed method we use the Singular value decomposition it is well known that the images, often used in variety of computer applications, are difficult to store and transmit. One possible solution to overcome this problem is to use a data compression technique where an image is viewed as a matrix and then the operations are performed on the matrix. In this paper, image compression is achieved by using singular value decomposition (SVD) technique on the image matrix. The advantage of using the SVD is the property of energy compaction and its ability to adapt to the local statistical variations of an image. Further, the SVD can be performed on any arbitrary, square, reversible and non reversible matrix of $m \times n$ size. In this paper, SVD is utilized to compress and reduce the storage space of an image. In addition, the paper investigates the effect of rank in SVD decomposition to measure the quality in terms of compression ratio and PSNR.

Index terms: Image compression, SVD (Singular Value Decomposition), De blocking filter, Blocking artifact, Video image.
\end{abstract}

\section{Introduction}

Image and video coding are one of the most important topics in image processing and digital communications. During the last thirty years we have witnessed a tremendous explosion in research a Applications in the visual communications field. There is no doubt that the beginning of the new century Revolves around the information society."Technologically speaking, the information society will be driven by audio and visual applications that allow instant access to multimedia information. This technological success would not be possible without image and video compression

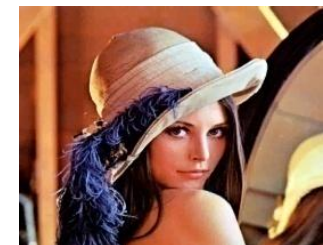

Figure 1: Input Video image

A video clip contains a series of still images (frames). Hence, to compress a video clips we can directly apply still image compression on each of these frames. In fact, this is the technique used by the "motion JPEG" standard. Although modern vide-compression algorithms go beyond still-image compression techniques and take into account similarities between frames to achieve further compression, they also employ the techniques used in still-image compression. Therefore, we begin our discussion by explaining the inner working of transform based digital-image Compression like JPEG.Impact of Digitization, Sampling and Quantization on Compression Generating Large number of bits; impacts storage and transmission Image limitations/video is correlated Human Visual System has limitations Types of Redundancies Spatial - Correlation between neighboring pixel values, Spectral - Correlation between different color planes or spectral bands, Temporal Correlation between different frames in a video sequence in this video image the lossless and lossy image compression the lossless image compression is Numerically identical to the original content on a pixel-by-pixel basis Motion Compensation is not used in lossless compression applications of lossless compression applications of lossless compression Medical Imaging, Contribution video applications the techniques of lossless compression is Bit Plane Coding, Lossless Predictive Coding DPCM, Huffman Coding of Differential Frames, Arithmetic Coding of Differential Frames the bit plane coding is a video frame with NxN pixels and each pixel is encoded by " $\mathrm{K}$ " bits Converts this frame into $\mathrm{K} \times(\mathrm{NxN})$ binary frames and encode each binary frame independently. Runlength Encoding, Gray Coding, Arithmetic coding.. 


\section{A)Video coding:}

During the last ten years, the hybrid schemecombining motion compensated prediction and DCThas represented the state of the art in video coding.This approach is used by the ITU H.261 and H.263 standards as well as for the MPEG-1 and MPEG-2 standards. However in 1993, the need to add new content-based functionalities and to provide the userthe possibility to manipulate the audio-visual content was recognized and a new standard effort known as MPEG-4 was launched. In addition to these functionalities, MPEG-4 provides also the possibility of combining natural and synthetic content. MPEG-4 phase 1 became an international standard in 1999 [3].MPEG-4 is having difficulties finding wide-spread use, mainly due to the protection of intellectual property and to the need to develop automatic and efficient segmentation schemes.The frame-based part of MPEG-4 which in corporate serror resilience tools, is finding its way in the mobile communications and Internet streaming. H.263, and several variants of it [6], are also very much used in mobile communication and streaming and it will be interesting to see how these two standards compete in these applications. The natural video part of MPEG-4 is also based in motion compensation prediction followed by the DCT, the fundamental difference is that of adding the coding of the object shape. Due to its powerful object-based approach, the use of the most efficient coding techniques, and the large variety of data types that it incorporates, MPEG-4 represents today the state-of-the-art in terms of visual data coding technology [5]. How MPEG-4 is deployed and what applications will make use of its many functionalities is still an open question. applications where the content provider wishes the user to obtain both the encoder and decoder from them so that the provider can gain economic advantage.All the video coding standards based on motion prediction and the DCT produce block artifacts at low data rate. There has been a lot of work using post-processing techniques to reduce blocking artifacts[1,2,3]. A great deal of work has been done to investigate the use of wavelets in video coding. This work has taken mainly two directions. The first one is to code the prediction error of the hybrid scheme using the DWT [4]. The second one is to use a full 3-D wavelet decomposition [5, 6]. Although these approaches have reported coding efficiency improvements with respect to the hybrid schemes, most of them are intended to provide further functionalities such as scalability and progressive transmission. One of the approaches that reports major improve-ments using the hybrid approach is the one proposed in [7]. Long-term memory prediction extendsmotion compensation from the previous frame to several past frames with the result of increased coding efficiency. The approach is combined with affine motion compensation. Data rate savings between 20 and 50\% are achieved using the test model of H.263+. The corresponding gains in PSNR are between 0.8 and $3 \mathrm{~dB}$. It can be said that MPEG-4 and H.263+ represent the state of the art in video coding. H.263+ provides a framework for doing frame-based low to moderate data rate robust compression. MPEG-4 combines frame-based and segmentation-based approaches along with the mixing of natural and synthetic content allowing efficient coding as well as content access and manipulation. There is no doubt that other schemes may improve the coding efficiency established in MPEG-4 and H.263+ but no significant breakthrough has been presented to date.

\section{Methodology}

The objective of this project is to apply linear algebra [?"Singular Value Decomposition (SVD)]"to midlevel image processing, especially to area of image compression and recognition. The method is factoring a matrix A into three new matrices $\mathrm{U}, \mathrm{S}$, and $\mathrm{V}$, in such way that $\mathrm{A}=\mathrm{USV}^{\mathrm{T}}$ Where $\mathrm{U}$ and $\mathrm{V}$ are orthogonal matrices and $\mathrm{S}$ is a diagonal matrix.

The experiments are conducted under different term $\mathrm{k}$ of singular value, and the outer product expansion of image matrix A for image compression. this project also demonstrates how to use SVD approach for image processing in area of Image Compression (IC)

\section{A) Properties of The SVD}

There are many properties and attributes of SVD, here we just present parts of the properties that we used in this project.

1. The singular value $\sigma_{1}, \sigma_{2}, \ldots \ldots \sigma_{\mathrm{n}}$ are unique, however, the matrices $\mathrm{U}$ and $\mathrm{V}$ are not unique.

2. Since $A A^{T}=V S^{T} S V^{T}$, so $V$ diagonalizes, it follows that the $v j \mathrm{~s}$ are the eigenvector of $A A^{T}$

3. Since $A A^{T}=U_{S S}{ }^{T} U^{T}$, so it follows that $U$ diagonalizes $A A^{T}$ and that the $U_{i}$ 's are the eigenvectors of $A A^{T}$.

4. If $A$ has rank of $r$ then $V_{j}, V_{j} \ldots V_{r}$. form an Ortho mal basis for range space of $A^{T} A T, R A^{T}$, and $U_{j}, U_{j}, \ldots .$. 践 form an orthonormal basis for .range space $A, R(A)$.

5. The rank of matrix $\mathrm{A}$ is equal to the number of its nonzero singular values

\section{B) SVD Approach for Image Compression}

Image compression deals with the problem of reducing the amount of data required to represent a digital image. Compression is achieved by the removal of three basic data redundancies: 1) coding redundancy, which is present when less than optimal. 2) interpixel redundancy, which results from correlations between the pixels. 
3) psychovisual redundancies, which is due to data that is ignored by the human visual [2].The property 5 of SVD in section 2 tells us the rank of matrix A is equal to the number of its nonzero singular values?h. In many applications,the singular values of a matrix decrease quickly with increasing rank. This propriety allows us to reduce the noise or compress the matrix data by eliminating the small singular values or the higher ranks. When an image is SVD transformed, it is not compressed, but the data take a formation. With this, we can use only a few singular values to represent the image with little differences from the original.To illustrate the SVD image compression process, we show detail procedures:

$$
\begin{aligned}
& \mathrm{A}=\mathrm{USV}^{\mathrm{T}}=\sum_{\mathrm{i}=1}^{\mathrm{r}} \sigma_{\mathrm{i}} \mathrm{u}_{\mathrm{i}} \mathrm{v}_{\mathrm{i}} \quad(1) \\
& \text { That is } \mathrm{A} \text { can be represented by the outer product expansion: } \\
& \mathrm{A}=\sigma_{1} \mathrm{u}_{1} \mathrm{v}_{1}^{\mathrm{T}}+\sigma_{2} \mathrm{u}_{2} \mathrm{v}_{2}^{\mathrm{T}}+\ldots \ldots \ldots+\sigma_{\mathrm{r}} \mathrm{u}_{\mathrm{r}} \mathrm{v}_{\mathrm{r}}^{\mathrm{T}} \quad \text { (2) }
\end{aligned}
$$

When compressing the image, the sum is not performed to the very last SVs, the SVs with small enough values are dropped. (Remember that the SVs are ordered on the diagonal.)The closet matrix of rank k is obtained by truncating those sums after the first $\mathrm{k}$ terms:

$$
A_{k=} \sigma_{1} u_{1} v_{1}^{T}+\sigma_{2} u_{2} v_{2}^{T}+\ldots \ldots \ldots+\sigma_{k} u_{k}
$$

The total storage for $\mathrm{k} A$ will be

$$
\mathrm{k}(\mathrm{m}+\mathrm{n}+1) \quad(4)
$$

The integer $\mathrm{k}$ can be chosen confidently less then $\mathrm{n}$, and the digital image corresponding to $\mathrm{k}$ A still have very close the original image. However, the chose the different $\mathrm{k}$ will have a different corresponding image and storage for it. For typical choices of the k, the storage required for $\mathrm{k}$ A will be less the 20 percentage.

\section{C) Image Compression Measures}

To measure the performance of the SVD image compression method, we can computer the compression factor and the quality of the compressed image. Image compression factorcan be computed using the Compression ratio:

$$
\mathrm{CR}=\mathrm{m} * \frac{\mathrm{n}}{\mathrm{k}(\mathrm{m}+\mathrm{n}+1)} \ldots \text { (5) }
$$

To measure the quality between original image $\mathrm{A}$ and the compressed image $\mathrm{k} A$, the

$$
\mathrm{MSE}=\frac{1}{\mathrm{mn}} \sum_{\mathrm{y}=1}^{\mathrm{m}} \mathrm{x} \sum_{\mathrm{x}=1}^{\mathrm{n}}\left(\mathrm{f}_{\mathrm{A}}(\mathrm{x}, \mathrm{y})-\mathrm{f}_{\mathrm{A} / \mathrm{c}}(\mathrm{x}, \mathrm{y}) \ldots\right.
$$

\section{New Trends In Image And Video Compression} cheapand

Before going any further, the following question has to be raised: if digital storage is becoming so

\begin{tabular}{|l|l|l|}
\hline Coding Generation & Approach & Technique \\
\hline Oth Generation & $\begin{array}{l}\text { Direct wave form } \\
\text { coding }\end{array}$ & PCM \\
\hline $1^{\text {st }}$ generation & $\begin{array}{l}\text { Redundancy } \\
\text { removal }\end{array}$ & $\begin{array}{l}\text { DPCM,DCT,DWT } \\
\text { DWT,VQ }\end{array}$ \\
\hline $2^{\text {nd }}$ generation & $\begin{array}{l}\text { Coding by } \\
\text { structure }\end{array}$ & Image segmentation \\
\hline $3^{\text {rd }}$ generation & $\begin{array}{l}\text { Analysis and } \\
\text { synthesis }\end{array}$ & Model based coding \\
\hline $4^{\text {th }}$ generation & $\begin{array}{l}\text { Recognition and } \\
\text { reconstruction }\end{array}$ & $\begin{array}{l}\text { Knowledge based } \\
\text { coding }\end{array}$ \\
\hline $5^{\text {th }}$ generation & Intelligent coding & Schematic coding \\
\hline
\end{tabular}

Table 1: image and video coding classification

\section{A) Image and video coding classification}

In order to have a broad perspective, it is important to understand the sequence of image and video coding developments expressed in terms of "generation based coding approaches. tabel 1 shows this classification according to [20].It can be seen from this classification that the coding community has reached third generation image and video coding techniques. MPEG-4 provides segmentation- based approaches as well as model based video coding in the facial animation part of the standard.

\section{B) Coding through merging of natural and synthetic content}

In addition to the use of metadata, future video coding schemes will merge natural and synthetic content. This will allow an explosion of new applications combining these two types of contents. MPEG-4 has provided a first step towards this combination by providing efficient ways of face encoding and animation. 
However, more complex structures are needed to model, code, and animate any kind of object.. Speech and audio will come to the rescue ofvideo (or viceversa) by combining both fields in an intelligent way. [8].

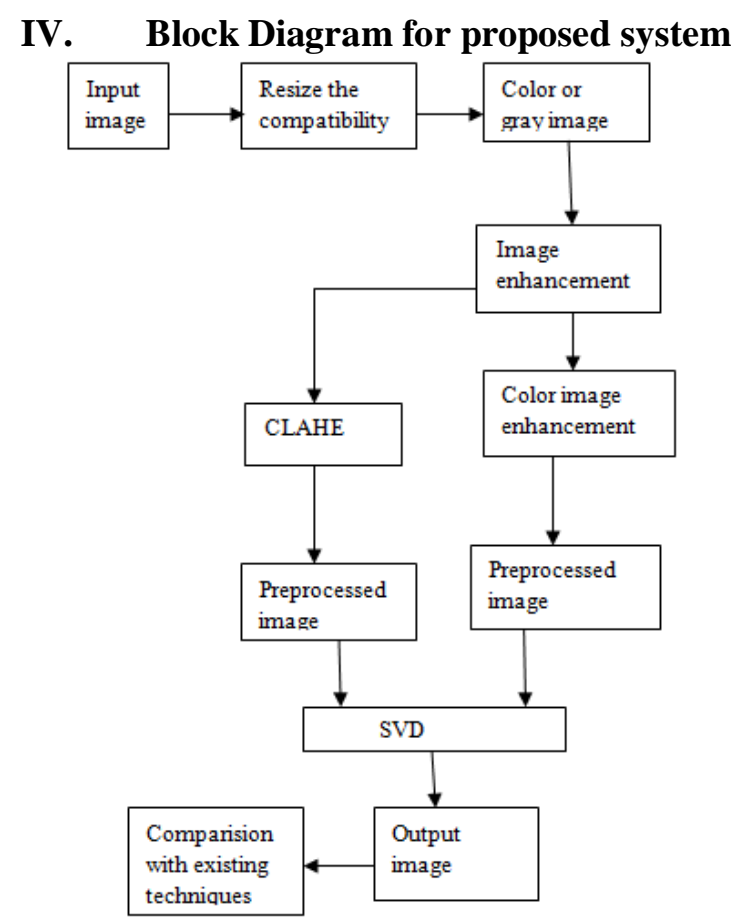

\section{A) Input Image}

Figure 2 :shows the schematic diagram of Proposed System Image.

The Figure 3 shows the input image. The input image is a gray image or color image. It is taken from video

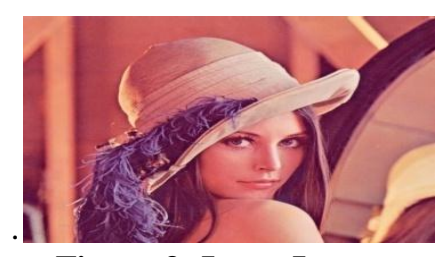

Figure 3: Input Image

The default size is about $576 * 768$.for our convinence the input image is resized ino512*512

B) Resize The Compatibility

It's having high stability of the image compression and the resize of the compatibility the image size is reduced.

\section{C) Color Image Enhanceemaent}

Dynamic Range of Intensity values Limited number of bit-planes varying illumination ver the image space.

- Brightness

- Contrast

- color

D) Pre Processing

Image pre-processing can significantly increase the reliability of an optical inspection. Several filter operations which intensify or reduce certain image details enable an easier or faster evaluation. Users are able to optimize a camera image

\section{E) CLAHE}

CLAHE (Contrast Limiting Adaptive Histogram Equalization) differ from ordinary Adaptive Histogram Equalization in its contrast limiting. CLAHE(Contrast Limiting Adaptive Histogram Equalization) was developed to prevent the over amplification of noise that adaptive histogram equalization can give rise to .This is achieved by limiting the contrast enhancement of AHE(Adaptive Histogram Equalization).Noise can be reduced while maintaining the high spatial frequency content of the image by applying a CLAHE(Contrast Limiting Adaptive Histogram Equalization). 


\section{F) COLOR IMAGE OR GRAY IMAGE}

The input and output image it may be gray image or color image it' $s$ for our convenience.

\section{G) HISOGRAM}

An image histogram is a type of histogram that acts as a graphical representation of the tonal distribution in a digital image. It plots the number of pixels for each tonal value. By looking at the histogram for a specific image a viewer will be able to judge the entire tonal distribution at a glance.

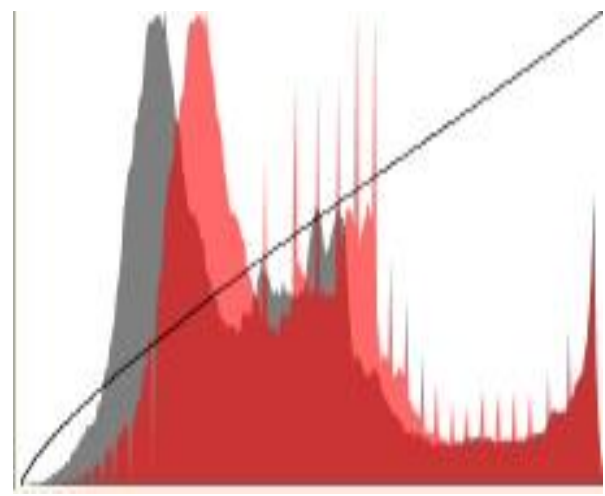

Figure4: Histogram Image

Histogram modeling techniques (e.g. histogram equalization) provide a sophisticated method for modifying the dynamic range and contrast of an image. The Figure7 shows the histogram image.

The horizontal axis of the graph represents the tonal variations, while the vertical axis represents the number of pixels in that particular tone. The left side of the horizontal axis represents the black and dark areas, the middle represents medium grey and the right hand side represents light and pure white areas. The vertical axis represents the size of the area that is captured in each one of these zones. Image histograms can be useful tools for thresholding. Because the information contained in the graph is a representation of pixel distribution as a function of tonal variation, image histograms can be analyzed for peaks and/or valleys which can then be used to determine a threshold value. This threshold value can then be used for edge detection, image segmentation, and co-occurrence matrices.

\section{H) Comparision With Existing Techniques :}

The comparision of the DCT to the output image table 2

\begin{tabular}{|l|l|l|l|}
\hline Bit savings\% & \multicolumn{3}{|c|}{ PSNR(db) } \\
\hline Books & 42 & 38 & 34 \\
\hline Watch & 5.0 & 6.4 & 3.2 \\
\hline Pen & 4.1 & 9.5 & 3.8 \\
\hline Mobile & 8.4 & 8.7 & 7.5 \\
\hline Flower & 11.2 & 6.5 & 9.7 \\
\hline Car & 9.5 & 4.3 & 1.8 \\
\hline Bus & 2.3 & 9.6 & 7.5 \\
\hline Glasses & 7.4 & 5.6 & 8.5 \\
\hline
\end{tabular}

We have, in fact, established that the performance of the integer versions is generally hardly distinguishable from the floating-point version of the same transform. Hence, from now on, to avoid unnecessary clutter in the presentation of the remainder of the experiments, we will only show results for the original floating-point transforms. More simulation results of test sequences at the QCIF resolution are shown in Table 2, where the operating points are chosen as 42,38 , and $34 \mathrm{~dB}$ of PSNR, and the coding performance is evaluated in terms of relative bit savings.

\section{Results And Discussion}

Outputs are above mentioned SVD(Singular Value Decomposition was simulated using MATLAB and the output is also shown

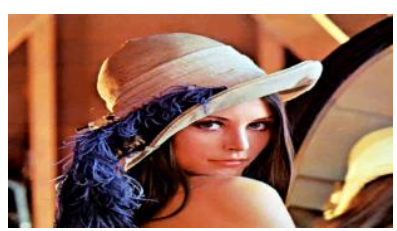

Figure5:Input image 
Figure 5 shows the input of the video image The video image is a gray image or color image . It is of default size $576 * 768$. For our convenience, the input image is resized to $512 * 512$.

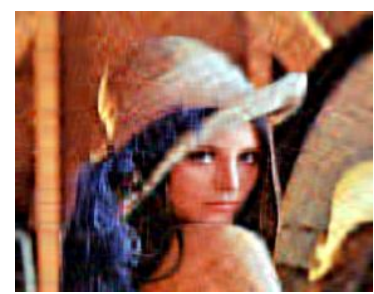

Figure 6: Output image

The Figure 6 shows the Output image, the output image is compressed to the input image. The file size is decreased to the input image the size is about approximately 30,000

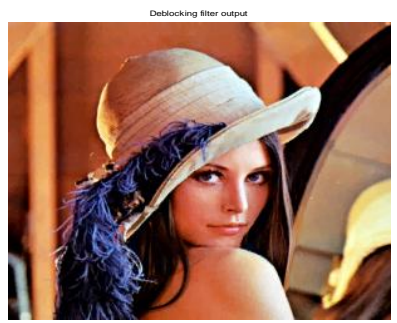

Figure 7: Deblocking Filter

The Figure7 shows that deblocking filter, A deblocking filter is a video filter applied to blocks in decoded video to improve visual quality and prediction performance by smoothing the sharp edges which can form between macro block.

\section{Conclusion:}

In this proposed method to reduce time complexity and the file size is decreased in image compression. The concept of SVD is a good compression ratio it has many practical and theoretical value other than image compression. The proposed algorithm uses the image can be very useful to save the storage. The result obtained for image compressing has satisfactory of image compression ratio compare with image quality; the results for image compression with a small error percentage compare with using the original image dimensions.

\section{References}

[1]. Jingning Han,Ankur Saxena, Vinay Meloke, and Kenneth Rose "Jointly Optimal spatial prediction and Block transform for videoand image coding," IEEE Trans on image processing.vol.21.no.4.April.201

[2]. Han.J, Saxena .A, and Rose .K, "Towards jointly optimal spatial prediction and adaptive transform in video/image coding," in Proc. IEEE ICASSP, Mar. 2010, pp. 726-729.

[3]. Xu .K, Wu .K, and Zhang.W, "Intra-predictive transforms for block based image coding," IEEE Trans. Signal Process., vol. 57, no. 8, pp. 3030-3040, Aug. 2009.

[4]. Ye .Y and Karczewicz .M, "Improved H.264 intra coding based on bi-directional intra prediction, directional transform, and adaptive coefficient scanning," in Proc. IEEE ICIP, Oct. 2008, pp. 2116-2119.

[5]. Zeng .B and Fu .J, "Directional discrete cosine transforms-A new framework for image coding," IEEE Trans. Circuits Syst. Video Technol., vol. 18, no. 3, pp. 305-313, Mar. 2008.

[6]. Yueh.W. C, "Eigen values of several tridiagonalmatrices," Appl.Math. E-Notes, vol. 5, pp. 66-74, Apr. 2005.

[7]. Wiegand .T, Sullivan .G. J, Bjontegaard .G, and Luthra .A, "Overview of the H.264/AVC video coding standard," IEEE Trans. Circuits Syst.Video Technol., vol. 13, no. 7, pp. 560-576, Jul. 2003.

[8]. Malvar. H. S, Hallapuro. A, Karczewicz. M, and Kerensky .L, "Low complexity transform and quantization in H.264/AVC," IEEE Trans. Circuits Syst. Video Technol., vol. 13, no. 7, pp. 598-603, Jul. 2003.

[9]. Marpe .D, George .V, Cycon. H. L, and Barthel. K. U, "Performance evaluation of Motion-JPEG2000 in comparison with H.264/AVC operated in pure intra coding mode," in Proc. SPIE, Oct. 2003, vol. 5266, pp. 129-137.

[10]. Strang.G, "The discrete cosine transform," SIAM Rev., vol. 41, no. 1,pp. 135-147, Mar. 1999.

[11]. Sikora. T and $\mathrm{Li}$.H, "Optimal block-overlapping synthesis transforms for coding images and video at very low bitrates," IEEE Trans. Circuits Syst. Video Technol., vol. 6, no. 2, pp. 157-167, Apr. 1996.

[12]. Rao. K. R and Yip .P, Discrete Cosine Transform-Algorithms, Advantages and Applications. New York: Academic, 1990.

[13]. Rose .K, Heiman .A, and Dinstein .I, “DCT/DST alternate-transform image coding,” IEEE Trans. Commun., vol. 38, no. 1, pp. 94101, Jan.1990. 